\title{
Immunosuppressive Consequences of Radiotherapy and Chemotherapy in Patients with Acute Lymphoblastic Leukaemia
}

\author{
A. C. CAMPBELL, P. HERSEY, I. C. M. MACLENNAN, H. E. M. KAY, M. C. PIKE, \\ and the MEDICAL RESEARCH COUNCIL'S WORKING PARTY ON LEUKAEMIA IN CHILDHOOD
}

British Medical fournal, 1973, 2, 385-388

\section{Summary}

Assays of lymphocyte subpopulations and function have been performed on patients with acute lymphoblastic leukaemia still in remission after 18 months' treatment. The patients were subjects of a trial of the value of craniospinal irradiation in the third month of treatment in preventing central nervous system relapse. Effects of both irradiation and chemotherapy were observed. Lymphopenia was much more marked in those patients who had received irradiation over a year previously. Assays of response to phytohaemagglutinin (P.H.A.) and staining for surface immunoglobulin indicated that this difference was due to a deficiency in thymusdependent lymphocytes in the irradiated children. Chemotherapy had a particularly marked depressant effect on lymphocytes with antibody-dependent cytotoxic activity and a proportion of PHA-responsive cells were also depressed. It may be relevant that four of the patients depleted of thymus-dependent lymphocytes by radiation died of infection during remission, while none of those treated with chemotherapy alone died in remission.

\section{Introduction}

Immunosuppression caused by agents used in the treatment of leukaemia may be important for two reasons. Firstly, the more the patient's immunological function is impaired the more susceptible to infection he is likely to be. The increased risk of fatal infections in patients treated with $x$-irradiation and cytotoxic drugs is well recognized. Secondly, true cure of leukaemia may be dependent on an effective immune response in the patient against his own neoplastic cells, or possibly against a causative agent. Attempts are continuing to treat leukaemia with active immunotherapy (Mathé, 1971; Crowther et al., 1973). If such treatment is to be adequately tested it will be important to know the relative immunosuppressive effects of different therapeutic regimens.

The recognition of functionally distinct subpopulations of human lymphocytes, and the development of test systems by which they may be quantitated, offers the possibility of more

*For composition of Working Party see accompanying paper, p. 381. precise quantitation of immunosuppression than was previously available. Such assays in vitro include the mitotic response to phytohaemagglutinin (PHA), believed to be a property of thymus-dependent lymphocytes ( $T$ cells), immunofluorescent staining for surface immunoglobulin which characterizes most if not all B cells, and an assay of the cytotoxic activity of lymphocytes against antibody-sensitized target cells. This type of cytotoxic activity appears to be mediated by lymphocytes whose development is independent of thymic influence (Harding et al., 1971; Campbell et al., 1972). These cells, however, are probably a separate subpopulation from those B lymphocytes which are the precursors of antibody-producing cells (Harding and MacLennan, 1972). We describe them as cytotoxic " $B$ " cells only because we are confident that they are not thymusdependent lymphocytes.

We have applied these three assays to the lymphocytes of patients with acute lymphoblastic leukaemia who have been treated in the trial of "prophylactic" radiotherapy which is reported in an accompanying paper (Medical Research Council, 1973). We describe the results obtained on patients who were still in first remission after 18 months' treatment. Follow-up studies on these patients are in progress to assess the changes occurring in lymphocyte subpopulations after this time, when the patients are being randomized to three groups: (1) to have no further treatment, (2) to continue with chemotherapy, and (3) to receive a short course of active immunotherapy. The results of these studies will be published at a later date.

\section{Patients and Methods}

All the children studied were patients in the trial of "prophylactic" central nervous system (C.N.S.) irradiation described in the accompanying paper (Medical Research Council, 1973). After the initial induction phase of treatment, patients in complete remission were divided into a group that received craniospinal irradiation and a group that received no C.N.S. "prophylaxis." Subsequently, all these patients were given identical cycles of chemotherapy, except that the irradiated patients were given intrathecal methotrexate during the first four 12-week cycles. Lymphocyte populations were assessed in those patients who were still in first remission at the end of the fifth 12-week cycle of chemotherapy (about 72 weeks after admission). All patients reaching this stage by 1 November 1972 were included in this study. Lymphocyte tests were carried out at the end of the fifth and the end of the sixth 12-week cycles of chemotherapy (fig. 1).

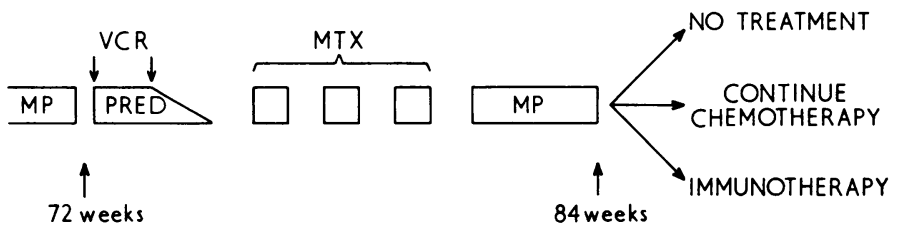

FIG. 1-Sixth cycle of chemotherapy. Lymphocyte assays were performed as indicated at end of fifth and sixth cycles. MP $=$ Mercaptopurine. MTX = Methotrexate. PRED =- Prednisolone. VCR = Vincristine. 


\section{LYMPHOCYTE ASSAYS}

More detailed descriptions of the assays of lymphocyte subpopulations are given elsewhere (Campbell et al., 1973). Absolute peripheral blood lymphocyte counts were obtained from the routine blood counts performed at the treatment centres. Samples of defibrinated venous blood were sent to Oxford by post for testing of lymphocytes. Most of the samples reached Oxford within 24 hours of collection; those that did not were discarded. In a large series of tests it has been found that storage of defibrinated blood for up to 24 hours at room temperature has only minor effects on the assays of lymphocyte function described here. All samples were tested between 18 and 24 hours after collection. Lymphocytes were harvested from the defibrinated blood by sedimentation at $37^{\circ} \mathrm{C}$ in gelatine $(3 \%$ in normal saline) (Coulson and Chalmers, 1964). After being washed in minimal essential medium and resuspended in RPMI 1640 plus $10 \%$ fetal bovine serum the mononuclear cells were counted by phase contrast microscopy.

\section{PHYTOHAEMAGGLUTININ STIMULATION}

Cultures were set up containing $5 \times 10^{5}$ mononuclear cells in $1 \mathrm{ml}$ of RPMI 1640 plus $10 \%$ fetal bovine serum with and without phytohaemagglutinin (PHA, Wellcome dried reagent) which was added to a concentration of $1: 1,000$. Whenever possible, tubes were set up in triplicate, but on occasion lymphocyte yields were low and fewer tubes had to suffice. The cells were incubated at $37^{\circ} \mathrm{C}$ for 72 hours, and the mitotic response was estimated as the incorporation of ${ }^{3} \mathrm{H}$-thymidine over a four-hour pulse between 68 and 72 hours. The PHA response is expressed as the counts per minute obtained in the stimulated cultures minus the counts per minute in the cultures without added PHA.

\section{STAINING FOR SURFACE IMMUNOGLOBULIN}

A modification of the membrane-staining technique described by Papamichail et al. (1971) was used. From the crude cell suspensions prepared by gelatine sedimentation, the lymphocytes were further purified by centrifugation on a ficoll-triosil gradient. The cells were washed and stained by incubation for 30 minutes at room temperature with fluorescein-conjugated sheep antihuman immunoglobulin (Wellcome). After washing to remove unbound conjugate the cells were examined by dark-ground fluorescent microscopy. Altogether, 200-300 cells were counted for each sample, and the percentage of cells staining for surface immunoglobulin was calculated.

\section{ANTIBODY-DEPENDENT CYTOTOXICITY}

The cytotoxic activity of lymphocytes against antibodysensitized target cells was measured by an isotope release technique using Chang human liver cells as the standard target cells (MacLennan, 1972). Suspensions of the mononuclear cells being tested were made up at three concentrations $(3.3 \times$ $10^{4}, 10^{5}$, and $3 \times 10^{5} / \mathrm{ml}$ ) in minimal essential medium plus $10 \%$ fetal bovine serum. Chang cells were labelled with ${ }^{51} \mathrm{Cr}$ chromate, washed, and made up to a suspension of $10^{4}$ cells $/ \mathrm{ml}$. Cultures were set up in triplicate containing $1 \mathrm{ml}$ of lymphocytes plus $1 \mathrm{ml}$ Chang cells. Anti-Chang antibody, a large pool of which has been prepared by immunization of rabbits, was added to a concentration of $1: 10,000$. Tubes were also set up containing Chang cells alone, without added lymphocytes. After incubation at $37^{\circ} \mathrm{C}$ for 20 hours the tubes were centrifuged and $1 \mathrm{ml}$ of supernatant medium was transferred to empty tubes. From counts of gamma radiation in the residue and supernatant tubes, the percentage release of ${ }^{51} \mathrm{Cr}$ label and the percentage lysis of target cells were calculated. The cytotoxic activity of individual lymphocyte samples was estimated as the number of lymphocytes required in culture to cause $50^{\circ}$ o target cell lysis, and expressed as the logarithm ${ }_{10}$ of this number (designated $\left.\mathrm{SC}_{50}\right)$.

\section{EXPRESSION OF RESULTS}

The results of all assays are plotted on a logarithmic scale for two reasons. Firstly, it has been found in a large series of experiments that the accumulated results of each of these tests in healthy subjects approximate to a normal distribution on a logarithmic scale. Secondly, by using the same scale in plotting the results of the different tests quantitative comparisons are easily made between them.

The three assays of lymphocyte subpopulations give results which are related to the proportion of different lymphocytes in any sample. In measuring immunosuppression it may be more important to obtain results related to the number of such lymphocytes per $\mathrm{ml}$ of blood. We have therefore taken into account the peripheral blood lymphocyte counts of the patients at the time the blood was taken for testing. Thus, from the PHA response the mitogenic capacity was calculated as $\log _{10}$ [(the lymphocyte count per $\mathrm{ml}$ of blood) $\times$ (the PHA response in counts per minute)]. The mitogenic capacity therefore represents the PHA responsiveness present in $1 \mathrm{ml}$ of patient's blood. From the assay of cytotoxic activity, the cytotoxic capacity is calculated as $\left[\log _{10}\right.$ (lymphocyte count per $\mathrm{ml}$ ) $\left.-\mathrm{SC}_{50}\right]$. With the assay of staining for surface immunoglobulin, it is possible to calculate directly the absolute number of staining cells per $\mathrm{ml}$ of patient's blood.

We have not so far tested the lymphocytes of a population of healthy children in the same age group as these patients ( 3 to 17 years). However, "control" ranges are indicated in the figures by central vertical bars. In figs. 2 and 3 these represent the extremes for lymphocyte and neutrophil counts between the ages of 3 and 17 (Geigy, 1970). In the remaining figures they represent the mean \pm 1 S.D. which we have obtained using the lymphocytes of healthy adults in a large series of experiments. We are aware that there may be major differences in lymphocyte function and subpopulations between children and adults. These "control" values are indicated only as reference points.

\section{Results}

Data on lymphocyte populations at the end of the fifth and sixth cycles of treatment were obtained for 57 patients, although not

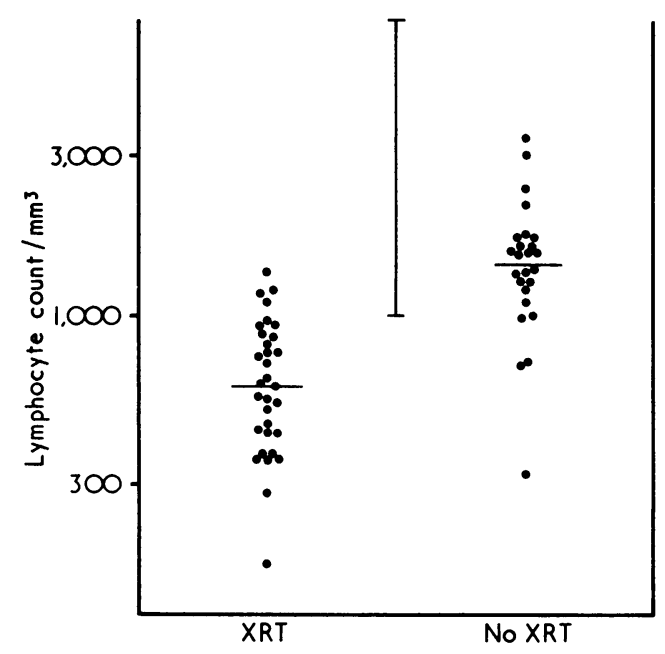

FIG. 2-Lymphocyte counts. As in all figures relating to lymphocyte assays, irradiated patients (XRT) are compared to non-irradiated (No XRT). A log. scale is used. Central bar represents extreme limits of lymphocyte counts for healthy children of same age range as patients. 
all of the tests were carried out on both these occasions. It was found that there were no significant differences between the pooled results for each assay system at these two times. The data have, therefore, been combined so that when results were available for a patient on both occasions the mean of the two was taken for analysis.

In each figure the combined data are compared for the two groups of patients, irradiated (XRT) and non-irradiated (No XRT).

Lymphocyte Counts. - The lymphocyte counts from children in both groups were below normal (fig. 2). In addition there is a striking difference between the irradiated and the non-irradiated

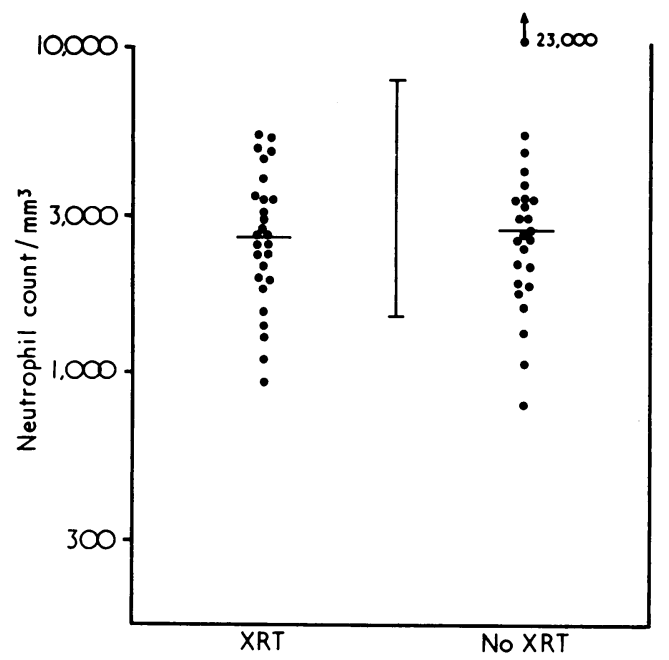

FIG. 3-Neutrophil counts. Details as for fig. 2 .

patients, the former group showing severe lymphopenia. This difference is not seen in other cells of the blood. For example, the neutrophil counts (fig. 3) are only moderately depressed, and this depression is equal in the two groups.

Lymphocyte Subpopulation Assays. - The much greater lymphopenia in the irradiated patients is reflected in the PHA response (fig. 4) which was significantly lower in this group than in the non-irradiated patients. The mitogenic capacity (fig. 4B) indicates the great difference in PHA responsiveness per $\mathrm{ml}$
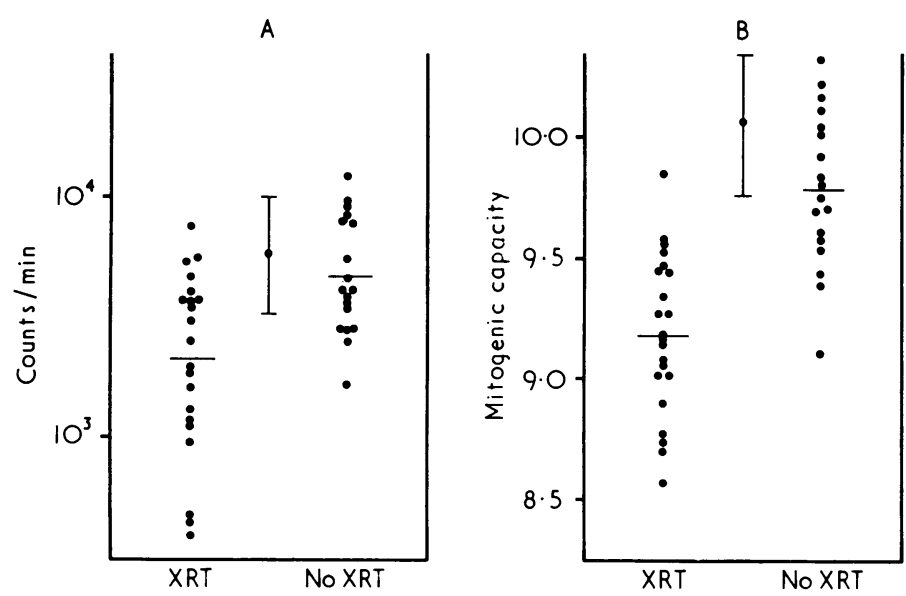

FIG. 4-A, Mitotic response to PHA. Incorporation of ${ }^{3} \mathrm{H}$-thymidine expressed as counts per minute. B, Mitogenic capacity of patient's blood. Central bar in figs. $4-6$ represents the mean \pm 1 S.D. for healthy adults.

of blood in the two groups of patients. Staining for surface immunoglobulin was carried out on fewer samples (fig. 5).
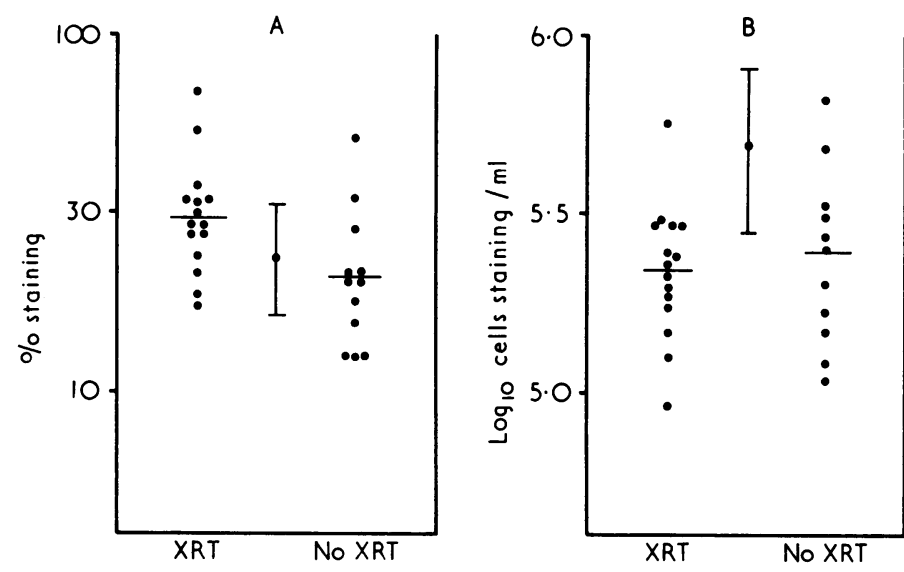

FIG. 5-A, Percentage of lymphocytes showing immunofluorescent stainin for immunoglobulin. B, Number of immunoglobulin staining cells per $\mathrm{ml}$ of blood.

Quite a different pattern was found-the irradiated patients having a higher percentage of staining lymphocytes than the non-irradiated patients. There is, however, no difference in the absolute number of staining cells per $\mathrm{ml}$ of blood between the two groups. The cytotoxic activity $\left(\mathrm{SC}_{50}\right)$ of lymphocytes from the irradiated patients was slightly higher than in the nonirradiated group (fig. 6A), although this difference is not
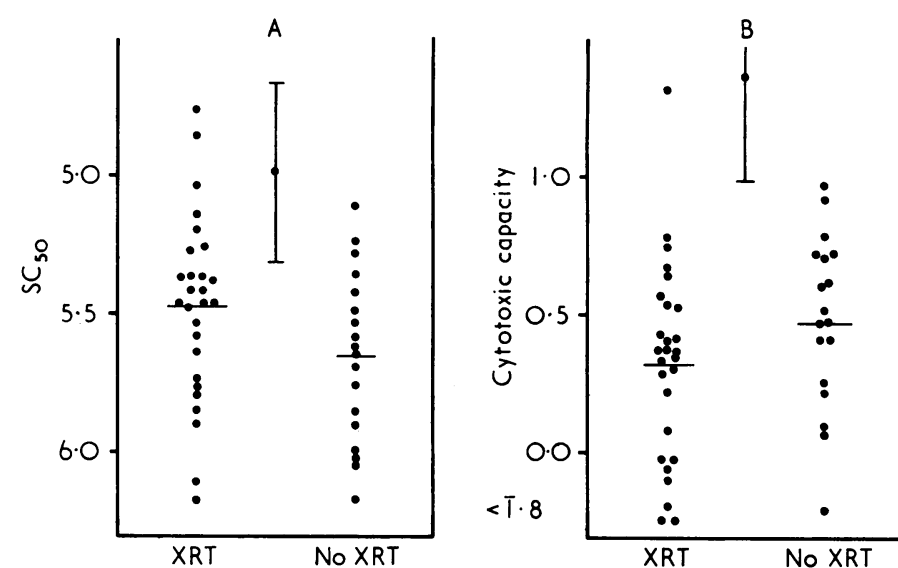

FIG. 6-A, Cytotoxic activity of patients' lymphocytes against antibody sensitized target cells, expressed as $\log _{10}$ of number of lymphocytes required to cause $50 \%$ target cell lysis $\left(\mathrm{SC}_{50}\right)$. B, Cytotoxic capacity of patients' blood.

statistically significant. When the blood lymphocyte count is allowed for, there is little difference in the mean circulating level of cytotoxic lymphocytes (cytotoxic capacity, fig. 6B). Both the $\mathrm{SC}_{50}$ and the cytotoxic capacity are greatly below the normal adult level in both groups of patients, suggesting particularly

Results of all Assays Compared for Irradiated and Non-irradiated Groups. Results are Expressed as Logarithmic Mean 1 S.D. Where Relevant, Actual Mean is Included in Parentheses

\begin{tabular}{|c|c|c|c|}
\hline & XRT & No XRT & $\mathrm{P}^{*}$ \\
\hline $\begin{array}{l}\text { Lymphocyte count } / \mathrm{mm}^{3} \\
\text { Neutrophil count } / \mathrm{mm}^{3} \\
\text { PHA response }(\text { count } /\end{array}$ & $\begin{array}{l}2 \cdot 780 \pm 0 \cdot 201(600) \\
3 \cdot 419 \pm 0 \cdot 209(2,620)\end{array}$ & $\mid \begin{array}{l}3 \cdot 147 \pm 0.209(1,400) \\
3 \cdot 449 \pm 0.273(2,810)\end{array}$ & $\begin{array}{c}<0.001 \\
\text { N.S. }\end{array}$ \\
\hline $\begin{array}{l}\text { min) } \\
\text { Mitogenic capacity } \cdots \\
\text { Percent. staining for Ig }\end{array}$ & $\begin{array}{l}3.335+0.354(2,160) \\
9.178+0.327 \\
1.472 \pm 0.159\left(29.6^{\prime \prime}{ }_{11}\right)\end{array}$ & $\left|\begin{array}{l}3.671 \pm 0.239(4,690) \\
9.783 \pm 0.320 \\
1.287 \pm 0.187\left(19 \cdot 4{ }^{\prime \prime}{ }_{0}\right)\end{array}\right|$ & $\begin{array}{l}0.004 \\
0.001 \\
0.01\end{array}$ \\
\hline 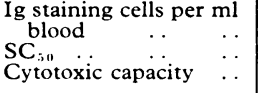 & $\begin{array}{l}5.324 \pm 0.184 \\
5.478 \pm 0.334 \\
0.323 \pm 0.358\end{array}$ & $\begin{array}{l}5.369 \pm 0.237 \\
5.654 \pm 0.308 \\
0.456 \pm 0.385\end{array}$ & $\begin{array}{l}\text { N.S. } \\
\text { N.S. (0.09) } \\
\text { N.S. }\end{array}$ \\
\hline
\end{tabular}

*P obtained by Wilcoxon test (Seigel, 1956)

N.S. = Not significant. 
pronounced suppression of this lymphocyte activity by the chemotherapy. The results of all these assays are summarized in the table.

\section{Discussion}

We have observed a number of changes in the blood lymphocytes resulting either from chemotherapy or craniospinal irradiation.

The particular sensitivity of lymphocytes to irradiation and the prolonged lymphopenia which is seen in patients after radiotherapy has often been described (Buckton et al., 1967; Stjernswärd et al., 1972). In this study it is strikingly apparent more than a year after irradiation. The mean lymphocyte count in the irradiated patients was $600 / \mathrm{mm}^{3}$ compared with $1,400 /$ $\mathrm{mm}^{3}$ in the patients treated with chemotherapy alone. Among the tests applied to the lymphocytes in vitro this difference is reflected in the level of lymphocytes responding to PHA. The distinct trend for the proportion of lymphocytes staining for surface immunoglobulin to be higher in the irradiated than in the non-irradiated children supports the conclusion that the low PHA responsiveness represents a deficiency of $T$ cells. These findings agree with those of Stjernswärd et al. (1972) who found that patients who had received radiotherapy for mammary carcinoma showed a prolonged loss of $T$ cells. In that study at least half of the thymus received a substantial dose of irradiation and it is possible that the deficiency of $T$ cells was a consequence of impaired thymic function. The children we have studied received irradiation to the spine, and therefore to a variable degree to the thymus. One of the factors controlling the dose to the thymus was the energy of the apparatus used which included, in different centres, supervoltage, $250 \mathrm{kV}$, and cobalt sources. We have not found any correlation between the energy of the irradiation source and the degree of depression of PHA response. This suggests that the loss of $T$ cells may not require irradiation of the thymus itself.

Since there was some variation in the total dose of methotrexate received by the two groups of patients (Medical Research Council, 1973) we have examined our data on this basis but find that the differences between the two groups are not related to dosage of this drug.

In assessing the effects that may be attributable to chemotherapy, the caution must be made that for the lymphocyte subpopulation tests we have only adult control levels with which to compare the patients. There is a depression of the peripheral blood lymphocyte count, which apparently involves all three subpopulations assayed. The lymphocyte assay showing greatest depression was that of cytotoxic activity against antibody-sensitized target cells. The mean cytotoxic capacity in these patients was almost tenfold below the normal adult level. Early results in the serial studies which we are continuing in these patients indicate that after stopping treatment with antileukaemic drugs there is a rapid rise in this lymphocyte subpopulation. We have also found in a group of patients with inflammatory bowel disease that treatment with azathioprine has a profound depressant effect on these cells (Campbell et al., 1973). Thus, the low levels of cytotoxic " $B$ " lymphocytes found in these patients may have been caused by mercaptopurine, a purine analogue chemically almost identical to azathioprine. The cytotoxic action of these lymphocytes in tumour rejection has been implicated in several animal models (Lamon et al., 1972; Hersey, 1973). They may be of similar significance in human neoplastic disease.

The depressive effect of irradiation on PHA-responsive T cells has been noted. A proportion of the PHA-responsive cells also appear to be sensitive to the chemotherapy used. In patients who have stopped drug treatment we have observed a rise in PHA response and mitogenic capacity over the following three to five weeks. This rise is seen in both the irradiated and nonirradiated patients, although the difference between these groups remains. This suggests that there may be two types of PHA-responsive cell, one long-lived and radiosensitive, the other short-lived and sensitive to cytotoxic agents such as mercaptopurine.

A positive correlation between our findings and the clinical results may lie in the observation that four patients in the irradiated group have died while in full remission, probably all as the result of infection. None of the non-irradiated patients have died in remission. It is possible that these deaths were the consequence of the immunosuppression, and more particularly the deficiency of long-lived $T$ lymphocytes, which resulted from $x$-irradiation. However, other possibilities remain. It may be that at an earlier stage the effect of irradiation on neutrophil production and function is of greater importance. Careful correlation with clinical and haematological findings will be necessary before the significance of changes in lymphocyte subpopulations can be assessed.

\section{Reference}

Buckton, K. E., Court Brown, W. M., and Smith, P. G. (1967). Nature, 214,

Campbell, A. C., MacLennan, I. C. M., Snaith, M. L., and Barnett, I. G. (1972). Clinical and Experimental Immunology, 12, 1 .

Campbell, A. C., Skinner, J., Hersey, P., MacLennan, I. C. M., and Truelove, S. C. (1973). In preparation.

Coulson, A. S., and Chalmers, D. G. (1964). Lancet, 1, 468.
Crowther, D. et al. (1973). British Medical fournal, 1, 131 .

Geigy (U.K.) Pharmaceuticals (1970). Geigy Scientific Tables. Macclesfield, Geigy.

Harding, B., and MacLennan, I. C. M. (1972). Immunology, 23, 35.

Harding, B., Pudifin, D. J., Gotch, Frances M., and MacLennan, I. C. M. (1971). Nature New Biology, 232, 80.

Hersey, P. (1973). In preparation.
Lamon, E. W., Skurzak, H. M., Klein, E., and Wigzell, H. (1972). fournal of Experimental Medicine, 136, 1072 .

MacLennan, I. C. M. (1972). Transplantation Reviews, 13, 67.

Mathe, G. (1971). Progress in Immunology, 1, 959

Medical Research Council (1973). British Medical fournal, 2, 381.

Papamichail, M., Brown, J. C., and Holborrow, E. J. (1971). Lancet, 2, 850 Siegel, S. (1956). Non-parametric Statistics, p. 152. London, McGraw-Hill. Stjernswärd, J., Joudal, M., Vanky, F., Wigzell, H., and Sealy, R. (1972). Lancet, 2, 1352. 